\title{
Constraining the merger history of Luminous Red Galaxies with SDSS and SALT
}

\author{
Rosalind E. Skelton* \\ South African Astronomical Observatory \\ 1 Observatory Road, Observatory, South Africa, 7925 \\ E-mail: rosesaao.ac.za \\ David G. Gilbank \\ South African Astronomical Observatory \\ 1 Observatory Road, Observatory, South Africa, 7925 \\ E-mail: gilbankesaao.ac.za

\section{Danièl N. Groenewald} \\ South African Astronomical Observatory \\ 1 Observatory Road, Observatory, South Africa, 7925 \\ E-mail: dgroenewald@saao.ac.za
}

\begin{abstract}
We present the preliminary results of a multi-semester campaign using the Robert Stobie Spectrograph on the Southern African Large Telescope (SALT) to study the mass growth of Luminous Red Galaxies (LRGs) at intermediate redshifts. We use SALT spectroscopy to measure the velocity difference between the LRGs and galaxies located within $50 \mathrm{kpc}$ of them, in order to determine what fraction of close pairs are physically related rather than projected pairs. SALT allows us to fill in an important region of parameter space by targeting LRGs with star-forming companions, for which there are very few spectroscopic measurements available in the public Sloan Digital Sky Survey (SDSS) data from which we draw our parent sample. We present the redshift measurements for 25 pairs of galaxies at $0.4<z<0.55$, finding that only 3 of the pairs are likely to be physically bound. This is much lower than the fraction of bound pairs in the SDSS sample, indicating that it will be essential to take the spectroscopic sample biases into account to determine the mass assembly history of LRGs.
\end{abstract}

SALT Science Conference 2015

1-5 June 2015

Stellenbosch Institute of Advanced Study, South Africa

\footnotetext{
*Speaker.
} 


\section{Introduction}

Luminous Red Galaxies (LRGs) are a key population of very massive galaxies. They are typically large elliptical galaxies, and are thought to be passively evolving, with very little ongoing star formation. It is not clear when and how the mass in these galaxies assembled, whether the majority of their mass formed in a single burst at high redshift or whether they grew more slowly through multiple mergers. There has been much debate over the last decade on how much LRGs have grown since $z=1$, a period of about 8 billion years [2, 5, 12]. In the standard hierarchical picture of galaxy formation, galaxies increase in mass by merging with nearby companions and accreting satellite galaxies. In most models of galaxy formation, mergers continue to be a particularly important channel of growth for LRGs down to the lowest redshifts. However, recent observational results show very little change in the distribution of galaxy masses since $z=1$ (e.g. $[14,9])$, suggesting that most LRGs were in place before this time and have experienced little or no recent growth.

The most direct approach for establishing whether LRGs are growing through mergers is to constrain the fraction of LRGs that have close companions, as a function of redshift, and estimate the relative change in mass expected if these companions merge with the LRG. By using close pairs of galaxies we avoid the subjectivity of identifying merger remnants as disturbed systems based on their morphology, and as the properties of the individual galaxies can be measured, we are able to estimate the mass in the companions, and place an upper limit on the mass growth through mergers.

In this work we have identified a large sample of LRGs at intermediate redshifts $(z \lesssim 0.5)$ and searched for nearby galaxies that are likely to merge with them. We use the publicly available Sloan Digital Sky Survey (SDSS, [15]) with spectroscopy from the SDSS-III Baryon Oscillation Spectroscopic Survey (BOSS, [7]) for the initial selection of LRGs. To confirm that pairs of galaxies are physically related, rather than projected pairs, one would ideally use spectroscopic redshifts to measure the difference in the velocities of the two galaxies in order to establish whether the pair is bound. While our LRGs are selected from a spectroscopic catalogue, their companions often do not have redshift measurements, making it difficult to assess the merger fraction. The BOSS spectrograph fibres cannot be placed very close together, restricting the likelihood that nearby companions will be targeted in the same observation. Strict targeting criteria prioritize galaxies that are likely to be massive and quiescent (ie. LRGs) based on their colours, creating a bias against bluer starforming companions. At intermediate redshifts we expect a significant fraction of galaxies to be star-forming, even in the relatively dense group environments in which LRGs are typically located. The contribution from these companions cannot be ignored. We therefore need to establish what fraction of companion galaxies are bound to the LRGs for an unbiased, mass-complete sample of companions to determine the true merger fraction. We have used SALT spectroscopy to follow up blue LRG companions that do not have spectroscopic measurements from SDSS, in order to fill in parameter space and create a representative sample from which to estimate the merger mass growth of LRGs.

In this conference proceedings we present the preliminary results of our spectroscopic programme using the Robert Stobie Spectrograph (RSS; [4]) on the Southern African Large Telescope (SALT; [3]). We present the spectra and measured redshifts for the sample in Section 2. In Section 3 we use the SALT data and the available spectroscopy from the SDSS to estimate the fraction of 
close companions that are bound to LRGs, finding large differences due to the biases in the sample selection. We discuss the implications of this and summarize in Section 4.

We assume a cosmology with $\Omega_{m}=0.3, \Omega_{\Lambda}=0.7$, and $H_{0}=70 \mathrm{~km} \mathrm{~s}^{-1} \mathrm{Mpc}^{-1}$ throughout. Magnitudes are on the AB system [10].

\section{Observations and data analysis}

We selected a large parent sample of LRGs from the $10^{\text {th }}$ Data Release of the SDSS [1] by querying the public SDSS database. ${ }^{1}$ LRGs are identified using strict magnitude and colour cuts to be targeted for spectroscopic follow up by BOSS [8]. Our full LRG sample covers a wide redshift range, from $0.2<z<0.7$, which will allow us to explore the evolution of the merger fraction and mass growth of LRGs over the second half of cosmic history. In this work we concentrate on the redshift range $0.4<z<0.55$ where we have used SALT to improve the spectroscopic coverage of galaxies near LRGs that are potential merger candidates. The spectroscopic completeness for galaxies close to LRGs drops dramatically at these redshifts, mainly due to the magnitude cuts of the SDSS and other follow-up spectroscopic surveys like Galaxy and Mass Assembly (GAMA, [13]). Even relatively small samples of galaxies with SALT redshift measurements will have a big impact, improving our ability to correct for the spectroscopic incompleteness of the full sample.

We selected LRGs identified as primary objects in the spectroscopic catalogues with a reliable redshift measurement (ZWARNING flag $=0$ ). Companion galaxies that may merge with the LRG in future were selected by querying the photometric catalogue database for neighbouring galaxies within $50 \mathrm{kpc}$ and within 1.5 magnitudes of each LRG in the r-band (a flux ratio of up to 1:4, corresponding to potential major mergers). We use the SDSS cModelMag as an estimate for the total magnitude of each galaxy throughout.

Observations of 27 pairs of galaxies were taken over the course of the 2014-1 and 20142 semesters as part of the a multi-semester programme 2014-1-MLT-002 (PI Skelton). Targets selected for follow-up spectroscopic observations with SALT are equatorial objects chosen from the parent SDSS sample to be visible to SALT. Fairly bright targets where the two galaxies had similar magnitudes were prioritized. We were able to successfully measure redshifts for both galaxies in the pair for 25 of the 27 observed pairs. The spectra and redshifts for 22 of these pairs are shown in Fig. 1. In addition to the magnitude and distance cuts described above, we particularly targeted companion galaxies with blue colours, in order to better sample the star-forming region of colour-magnitude space (see Fig. 2).

In each RSS observation we aligned the position angle of a long slit centred on the LRG such that the slit also covered the companion galaxy. This enables us to measure the relative velocity of the pair straightforwardly in a single observation. We used the PG0900 grating with a slit width of 2 arcseconds. Each observation consisted of two exposures of $1200 \mathrm{~s}$ each, with standard flats and arc calibrations taken after the science observation. We reduced the data following standard methods to bias subtract, correct for the gain and crosstalk, flat field and remove cosmic rays using a pipeline based on the PySALT spectroscopic data reduction tasks [6]. We used an automated routine to wavelength calibrate the reduced spectra (based on software written for the SAAO $1.9 \mathrm{~m}$ Cassegrain spectrograph by D. Gilbank).

\footnotetext{
${ }^{1}$ http://www.sdss3.org/dr10/
} 
Table 1: Galaxies targeted for SALT spectroscopy

\begin{tabular}{cccccc}
\hline \hline LRG Target ID & Companion SDSS ID & LRG $m_{r}[\mathrm{mag}]$ & Companion $m_{r}[\mathrm{mag}]$ & LRG z & Companion z \\
\hline J211611.24-003018.2 & 1237663542604334291 & 20.09 & 19.11 & 0.468 & 0.235 \\
J121314.36-015954.6 & 1237650762392011182 & 19.93 & 19.40 & 0.435 & 0.434 \\
J132731.03-003552.3 & 1237651503805825239 & 19.83 & 18.93 & 0.499 & 0.184 \\
J213022.77+000215.7 & 1237663457778860503 & 19.43 & 19.93 & 0.427 & 0.138 \\
J134625.15+000533.7 & 1237648704587235651 & 19.97 & 18.71 & 0.484 & 0.147 \\
J213313.02-000911.5 & 1237663543143039599 & 20.00 & 19.25 & 0.427 & 0.236 \\
J141503.12-003106.0 & 1237648720708633629 & 19.59 & 18.99 & 0.513 & 0.140 \\
J223329.74+000339.4 & 1237663479260971343 & 20.05 & 19.57 & 0.416 & 0.210 \\
J000351.17+004238.2 & 1237657191979352278 & 20.03 & 19.40 & 0.530 & 0.192 \\
J234530.80+002214.5 & 1237666408440857084 & 19.90 & 19.60 & 0.446 & 0.399 \\
J211921.60-002740.4 & 1237663542604662205 & 19.99 & 20.00 & 0.483 & 0.181 \\
J085239.68-004209.3 & 1237650795140547224 & 19.90 & 19.83 & 0.457 & 0.188 \\
J085246.03-001407.5 & 1237650795677483216 & 19.68 & 18.45 & 0.458 & 0.073 \\
J100114.36+001120.9 & 1237651801234211240 & 20.02 & 19.85 & 0.497 & 0.499 \\
J085953.03-000315.5 & 1237648721211031915 & 20.04 & 18.60 & 0.489 & 0.165 \\
J084032.18-002247.1 & 1237650795676107409 & 19.91 & 18.99 & 0.533 & 0.167 \\
J100454.14-011645.9 & 1237654872682267341 & 19.97 & 19.95 & 0.457 & 0.453 \\
J120752.49-011718.4 & 1237650372093411919 & 19.90 & 19.84 & 0.459 & 0.460 \\
J101258.75-004216.0 & 1237651800161780287 & 19.54 & 19.98 & 0.469 & 0.464 \\
J141503.12-003106.0 & 1237648720708633629 & 19.59 & 18.99 & 0.513 & 0.140 \\
J112626.09-004234.5 & 1237674649388122957 & 19.99 & 18.74 & 0.503 & 0.253 \\
J104612.79-000602.5 & 1237648721222631725 & 19.91 & 19.68 & 0.447 & 0.249 \\
J084917.92+001944.8 & 1237648721746723459 & 20.03 & 19.83 & 0.460 & 0.155 \\
J123054.06-013609.2 & 1237650762930782378 & 19.83 & 18.60 & 0.513 & 0.148 \\
J114411.93-011005.6 & 1237674648853217964 & 19.79 & 19.56 & 0.514 & 0.281 \\
\hline
\end{tabular}

Redshifts were measured by cross-correlating each spectrum with a star-forming and elliptical galaxy template spectrum using the IRAF EMSAO/XCSAO tasks. In most cases we coadded the one dimensional spectra from the two exposures to improve the $\mathrm{S} / \mathrm{N}$ before fitting redshifts, though in some cases the $\mathrm{S} / \mathrm{N}$ was high enough from a single exposure. Where we could measure redshifts from each individual exposure, the duplicate measurements provide a useful check of the quality of the redshift measurement. We also compared the LRG redshift measurements from SALT with those from BOSS, finding excellent agreement.

\section{Results}

The resulting redshifts for each LRG and companion are given in Table 1. In Fig. 1 we show the SALT spectra for 22 pairs of galaxies shifted to the rest-frame using the measured redshifts, as given in the top left hand corner of each panel. The LRG's spectrum is shown in black and the companion galaxy in blue.

Figure 2 shows the location of galaxies in our SALT sample in colour-magnitude space compared to the full parent sample of LRG pairs. The numbers of objects as a function of $g-r$ colour are shown by the histograms in the right hand panels. Grey symbols indicate LRGs (upper panels) and companion galaxies within $50 \mathrm{kpc}$ (lower panels) where there is no spectroscopic information 


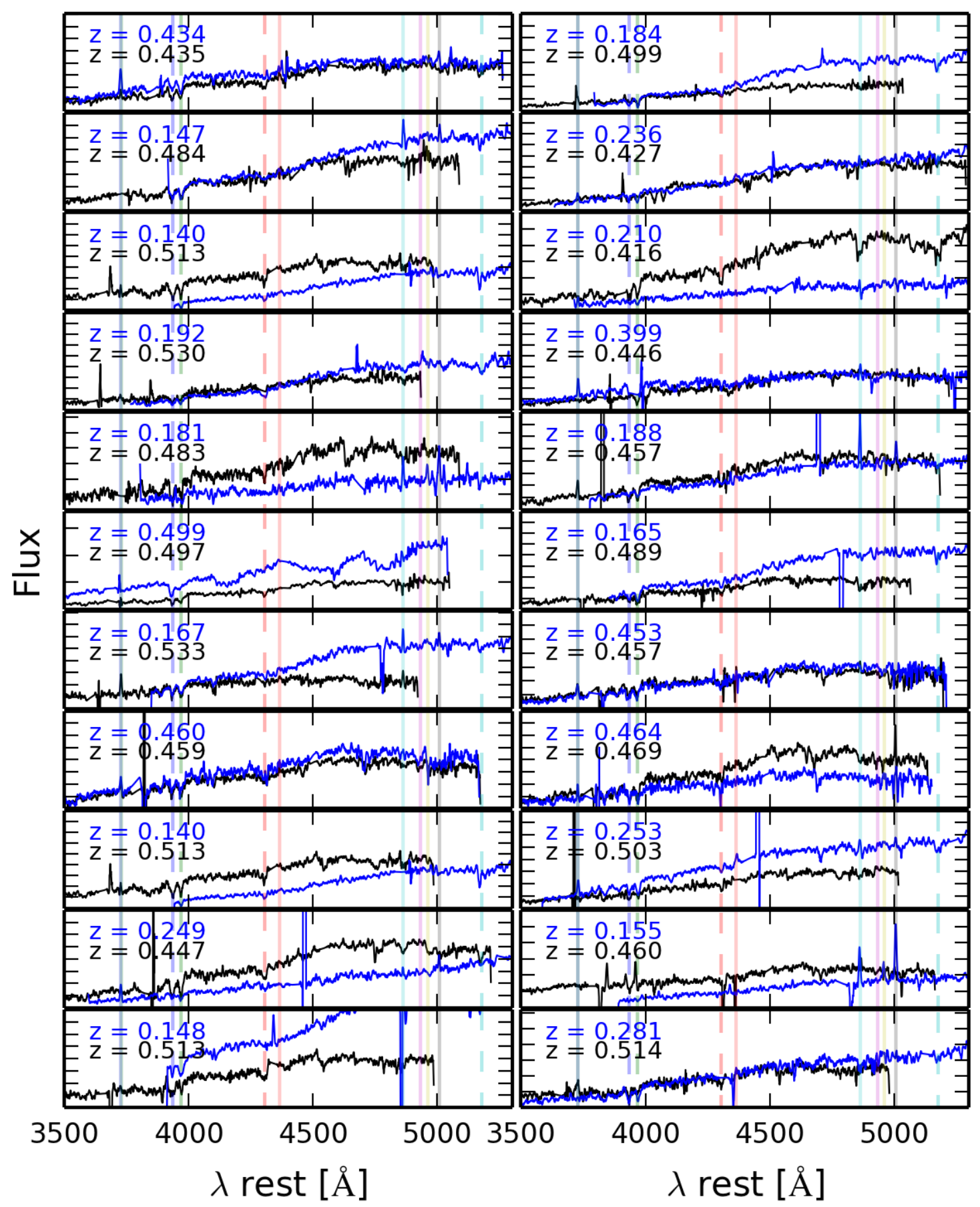

Figure 1: Spectra of 22 pairs of galaxies observed with SALT in the rest-frame (arbitrary flux scaling). In each panel the black line shows the spectrum of the LRG and the blue line the companion galaxy. The measured redshifts are given in the upper left hand corner of each panel. The positions of significant absorption (emission) features are indicated by the vertical dashed (solid) lines in each panel. 

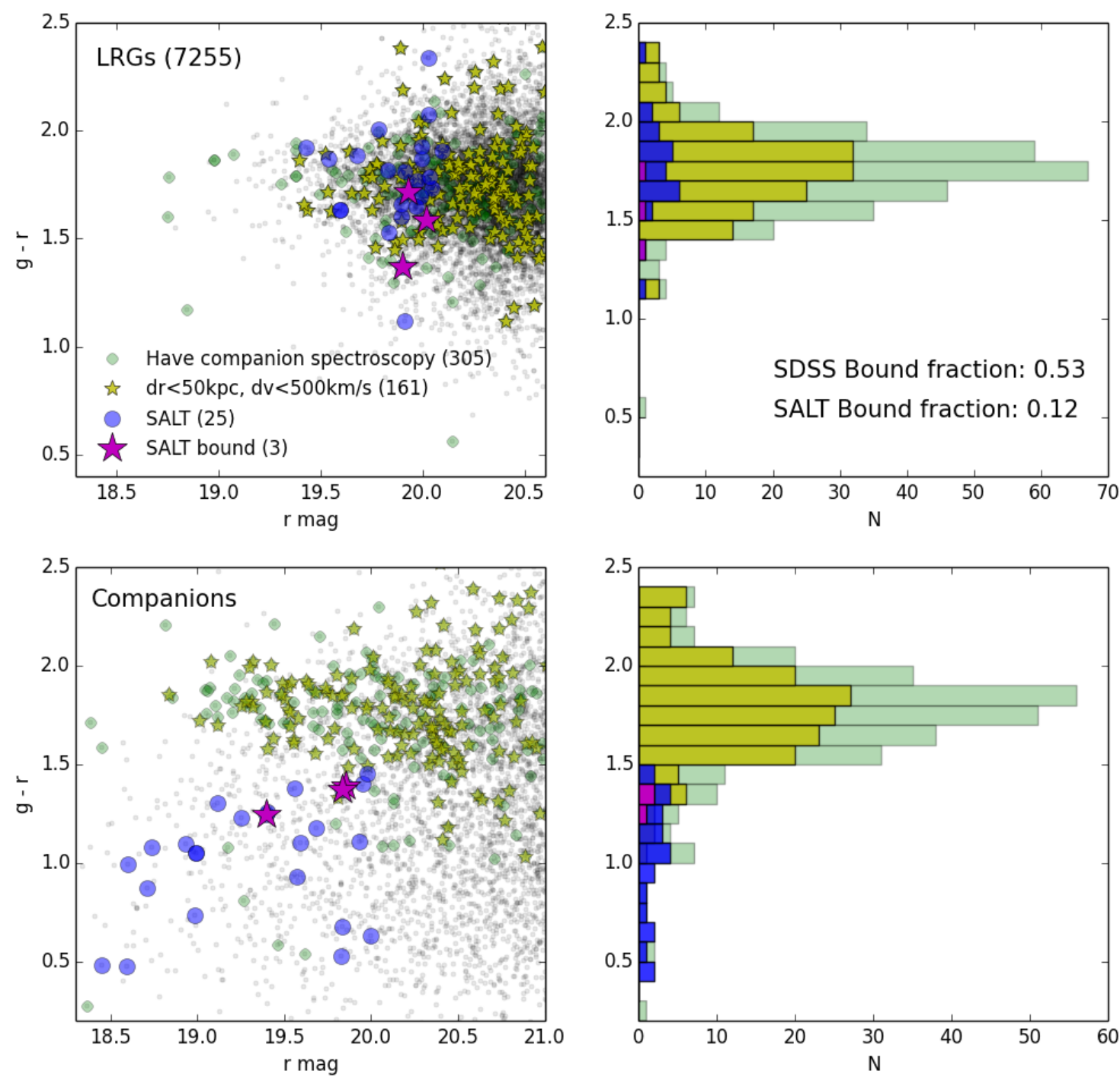

Figure 2: The distribution of LRGs (upper panels) and their companions (lower panels) in colour-magnitude space. The right hand panels show the number of objects with spectroscopic distance measurements as a function of colour. LRGs are red sequence galaxies by definition, however their companions have much wider range of colours. The full photometric sample is shown in grey. As targeting for SDSS spectroscopy (green and yellow symbols and histograms) is biased against blue galaxies, very few blue companions have SDSS redshift measurements. SALT observations (blue and purple points) fill in this gap. Yellow stars indicate pairs with SDSS spectroscopy that satisfy the criteria for being bound physical pairs. Purple stars indicate pairs of galaxies with SALT spectroscopy found to be at the same redshift and likely to be physically bound. The fraction of bound pairs is much lower in the blue companion sample (12\% vs. 53\%). 
for the companion galaxy. As there are many more objects without spectroscopy than with spectroscopy (7255 vs. 330), histograms of the full photometric sample are not shown. Colour can be used as a proxy for star formation rate. Most LRGs lie on the red sequence and are quiescent, with low levels of star formation. The companion distribution spans a much wider range in colour (more of them are star-forming). The SDSS spectroscopic sample (green and yellow points and histograms) is biased against blue galaxies, therefore the majority of galaxies with spectra lie on the red sequence, and companions in the blue cloud are unlikely to have redshift measurements.

Without spectroscopy it is not possible to distinguish physically bound pairs from projected pairs. The large symbols are galaxies which do have spectroscopic redshifts - green and yellow symbols are galaxies with SDSS spectroscopy, blue and purple symbols are the SALT sample. Using typical criteria in the literature on close pairs, if the velocity difference of the two galaxies in a pair is $\Delta v<500 \mathrm{~km} \mathrm{~s}^{-1}$ and the projected distance $r_{p}<50 \mathrm{kpc}$, we classify the pair as likely to be physically bound (e.g. [11]). The circles show unbound pairs that do not satisfy the velocity cut, and the stars show pairs that do satisfy this cut and are therefore likely to be physical pairs. From our preliminary redshift measurements, we find that in the SALT sample of 25 pairs, only 3 are bound. This is $12_{-4}^{+10} \%$ of the SALT sample. In comparison, for the sample of 305 pairs with SDSS spectroscopy, more than half are bound (161 pairs, or $53 \pm 3 \%$ ).

\section{Summary and future work}

We have used SALT RSS spectroscopy to improve the spectroscopic coverage of a sample of LRGs and their companion galaxies in close pairs at $0.4<z<0.55$. The publicly available spectroscopic data from SDSS is highly biased towards red galaxies. By including new data from SALT, we obtain a much more representative spectroscopic sample, improving coverage in the bright, blue region of colour-magnitude space. Using the SDSS spectroscopic data alone, approximately half of the LRGs in pairs appear to be physically bound to their companion galaxies (53 $\pm 3 \%)$. We find that in the SALT sample of 25 pairs, only 3 are bound $\left(12_{-4}^{+10} \%\right)$. The bound fraction for these blue, star-forming companions is much lower than for the biased SDSS spectroscopic sample, indicating that projection effects are likely to play a much more important role than previously expected. We will combine the SALT measurements with other available redshifts to carefully estimate the bound fraction, taking into account the inherent biases in the spectroscopic samples, in order to measure the merger fraction of LRGs and resultant growth from mergers over the last $5 \mathrm{Gyr}$ of cosmic history. SALT has enabled us to fill in an important yet under represented part of parameter space, with a relatively modest investment of time. We are now extending this work to explore the impact of the density of galaxies on the assembly history of massive red galaxies, using SALT RSS multi-object spectroscopy to obtain redshifts for multiple galaxies in the surroundings of LRGs in different environments.

\section{Acknowledgments}

This research is supported by a National Research Foundation Professional Development Programme grant. RES, DGG and DNG acknowledge financial support from the National Research Foundation. Funding for the Sloan Digital Sky Survey IV has been provided by the Alfred P. Sloan 
Foundation, the U.S. Department of Energy Office of Science, and the Participating Institutions. SDSS-IV acknowledges support and resources from the Center for High-Performance Computing at the University of Utah. The SDSS web site is www.sdss.org.

\section{References}

[1] Ahn, C. P., Alexandroff, R., Allende Prieto, C., et al., The Tenth Data Release of the Sloan Digital Sky Survey: First Spectroscopic Data from the SDSS-III Apache Point Observatory Galactic Evolution Experiment, ApJS, 211, 17 (2014)

[2] Brown, M. J. I., Dey, A., Jannuzi, B. T., et al., The Evolving Luminosity Function of Red Galaxies, ApJ, 654, 858 (2007)

[3] Buckley, D. A. H., Swart, G. P., \& Meiring, J. G., Completion and commissioning of the Southern African Large Telescope, in Society of Photo-Optical Instrumentation Engineers (SPIE) Conference Series, Vol. 6267, Society of Photo-Optical Instrumentation Engineers (SPIE) Conference Series, 0 (2006)

[4] Burgh, E. B., Nordsieck, K. H., Kobulnicky, H. A., et al., Prime Focus Imaging Spectrograph for the Southern African Large Telescope: optical design, in Society of Photo-Optical Instrumentation Engineers (SPIE) Conference Series, Vol. 4841, Instrument Design and Performance for Optical/Infrared Ground-based Telescopes, ed. M. Iye \& A. F. M. Moorwood, 1463-1471 (2003)

[5] Cimatti, A., Daddi, E., \& Renzini, A., Mass downsizing and "top-down" assembly of early-type galaxies, A\&A, 453, L29 (2006)

[6] Crawford, S. M., Still, M., Schellart, P., et al., PySALT: the SALT science pipeline, in Society of Photo-Optical Instrumentation Engineers (SPIE) Conference Series, Vol. 7737, Society of Photo-Optical Instrumentation Engineers (SPIE) Conference Series, 25 (2010)

[7] Dawson, K. S., Schlegel, D. J., Ahn, C. P., et al., The Baryon Oscillation Spectroscopic Survey of SDSS-III, AJ, 145, 10 (2013)

[8] Eisenstein, D. J., Annis, J., Gunn, J. E., et al., Spectroscopic Target Selection for the Sloan Digital Sky Survey: The Luminous Red Galaxy Sample, AJ, 122, 2267 (2001)

[9] Moustakas, J., Coil, A. L., Aird, J., et al., PRIMUS: Constraints on Star Formation Quenching and Galaxy Merging, and the Evolution of the Stellar Mass Function from $z=0-1$, ApJ, 767, 50 (2013)

[10] Oke, J. B., Absolute Spectral Energy Distributions for White Dwarfs, ApJS, 27, 21 (1974)

[11] Patton, D. R., Carlberg, R. G., Marzke, R. O., et al., New Techniques for Relating Dynamically Close Galaxy Pairs to Merger and Accretion Rates: Application to the Second Southern Sky Redshift Survey, ApJ, 536, 153 (2000)

[12] Robaina, A. R., Bell, E. F., van der Wel, A., et al., The Merger-driven Evolution of Massive Galaxies, ApJ, 719, 844 (2010)

[13] Robotham, A., Driver, S. P., Norberg, P., et al., Galaxy and Mass Assembly (GAMA): Optimal Tiling of Dense Surveys with a Multi-Object Spectrograph, PASA, 27, 76 (2010)

[14] Tomczak, A. R., Quadri, R. F., Tran, K.-V. H., et al.,Galaxy Stellar Mass Functions from ZFOURGE/CANDELS: An Excess of Low-mass Galaxies since $z=2$ and the Rapid Buildup of Quiescent Galaxies, ApJ, 783, 85 (2014)

[15] York, D. G., Adelman, J., Anderson, J. J. E., et al., The Sloan Digital Sky Survey: Technical Summary, AJ, 120, 1579 (2000) 